\title{
The influence of periodic islands in the flow on a scalar tracer in the presence of a steady source
}

\author{
M. R. Turner, J. Thuburn, and A. D. Gilbert \\ Mathematics Research Institute, School of Engineering, Computing and Mathematics, University of Exeter, \\ Exeter EX4 4QF, United Kingdom
}

(Received 19 January 2009; accepted 27 May 2009; published online 26 June 2009)

\begin{abstract}
In this paper we examine the influence of periodic islands within a time periodic chaotic flow on the evolution of a scalar tracer. The passive scalar tracer is injected into the flow field by means of a steady source term. We examine the distribution of the tracer once a periodic state is reached, in which the rate of injected scalar balances advection and diffusion with the molecular diffusion $\kappa$. We study the two-dimensional velocity field $\mathbf{u}(x, y, t)=2 \cos ^{2}(\omega t)(0, \sin x)+2 \sin ^{2}(\omega t)(\sin y, 0)$. As $\omega$ is reduced from an $O(1)$ value the flow alternates through a sequence of states which are either globally chaotic, or contain islands embedded in a chaotic sea. The evolution of the scalar is examined numerically using a semi-Lagrangian advection scheme. By time-averaging diagnostics measured from the scalar field we find that the time-averaged lengths of the scalar contours in the chaotic region grow like $\kappa^{-1 / 2}$ for small $\kappa$, for all values of $\omega$, while the behavior of the time-averaged maximum scalar value, $\overline{C_{\max }}$, for small $\kappa$ depends strongly on $\omega$. In the presence of islands $\overline{C_{\max }} \sim \kappa^{-\alpha}$ for some $\alpha$ between 0 and 1 and with $\kappa$ small, and we demonstrate that there is a correlation between $\alpha$ and the area of the periodic islands, at least for large $\omega$. The limit of small $\omega$ is studied by considering a flow field that switches from $\mathbf{u}=(0,2 \sin x)$ to $\mathbf{u}=(2 \sin y, 0)$ at periodic intervals. The small $\kappa$ limit for this flow is examined using the method of matched asymptotic expansions. Finally the role of islands in the flow is investigated by considering the time-averaged effective diffusion of the scalar field. This diagnostic can distinguish between regions where the scalar is well mixed and regions where the scalar builds up. (C) 2009 American Institute of Physics. [DOI: 10.1063/1.3159615]
\end{abstract}

\section{INTRODUCTION}

When a scalar tracer is injected into a fluid flow, the advection process can create fine scale structure in the tracer, which is subsequently destroyed by molecular diffusion. This advection-diffusion combination tends to homogenize the scalar and eventually the system evolves into a uniform steady state if there is no additional source of scalar. However, if additional scalar is injected into the flow via a steady source term, then, for a time periodic flow in a periodic domain, the system evolves into a periodic state, where scalar diagnostics oscillate about some time-averaged value. ${ }^{1,2}$ The efficiency of statistically stationary, homogeneous, isotropic flows (which include turbulence) to mix scalars was examined by Doering and Thiffeault. ${ }^{2}$ They calculate an upper bound for the scalar concentration for various source terms; however, the actual maximum concentrations are sensitive to the velocity field, and in particular the existence of periodic islands in the flow. Advection-diffusion problems with a source term are also important in the study of air pollution; ${ }^{3}$ thus understanding how much pollutant builds up in stagnant regions, such as islands, is very important. This study is therefore motivated by trying to understand better how scalar builds up in the presence of these islands, including the dependence on the size of the islands.

In the current study we examine the periodic states of time periodic flows by considering a scalar tracer which is injected steadily into an unsteady, two-dimensional, periodic velocity field. We choose the velocity field $\mathbf{u}(x, y, t)=2 \cos ^{2}(\omega t)(0, \sin x)+2 \sin ^{2}(\omega t)(\sin y, 0)$,

which, depending on the value of $\omega$, is either globally chaotic or consists of periodic islands embedded in a chaotic sea. This flow, which is a modification of the time-periodic sine flow (TPSF), is chosen so we can investigate how the periodic state is dependent on the islands. By varying the control parameter $\omega$ we can change the period of the flow and hence the size of the periodic islands, and for certain values of $\omega$ the islands disappear altogether. The evolution of scalar in this flow in the absence of a source term was studied by Cerbelli et $a l^{4}$ and Giona $e t a l{ }^{5}$ It is well known that periodic islands inhibit mixing by trapping scalar within them, ${ }^{6,7}$ but a thorough study investigating the quantity of scalar that builds up in islands in the presence of a source term has not been carried out to date. Gleeson ${ }^{7}$ studied the evolution of a strip of scalar placed inside a flow field for two initial conditions, one when the scalar crosses a periodic island, and one where it does not. The results show that the scalar initially within the island winds up and homogenizes, but stays within the island, while the other case shows that the scalar becomes well mixed in the surrounding chaotic sea without being transported into the periodic island. In this example a mixing measure $m(t)$ is defined, which in a "vortexlike mixing period" decays as $\mathrm{Pe}^{-\alpha}$, where Pe is the Péclet number (proportional to the inverse of the molecular diffusion) and $\alpha$ is a real scaling exponent. However, in the current study the presence of a steady source means that there will be a continuous injection of scalar both into the islands 
and the chaotic region. The work of Doering and Thiffeault ${ }^{2}$ confirmed that there will be an upper bound on the scalar value in the islands, but we will show a correlation between the time-averaged maximum scalar value in the islands and their size, for varying $\kappa$.

By considering time averages over one period of the flow field, we produce scalar diagnostics that are steady and hence are easier to analyze. We shall consider the evolution of both the time-averaged maximum scalar value $\overline{C_{\max }}$ and the time-averaged contour length, as functions of the molecular diffusion $\kappa$. The case of a slowly varying velocity field (small $\omega$ ) is examined analytically by studying the TPSF which has a velocity field that instantaneously switches between $\mathbf{u}=(0,2 \sin x)$ and $\mathbf{u}=(2 \sin y, 0)$ periodically with period $2 t_{s}$. By the use of matched asymptotic expansions we analytically determine how $C_{\max }$, and subsequently $\overline{C_{\max }}$, vary with $\kappa$.

As well as these diagnostics another useful measure is the effective diffusion of the scalar field. Transforming the advection-diffusion equation using a quasi-Lagrangian coordinate system ${ }^{8}$ based on the area inside scalar concentration contours $^{9,10}$ leads to a pure diffusion equation, where the diffusion coefficient is known as the effective diffusion. The effective diffusion is a useful diagnostic tool because it requires no prior knowledge of the velocity field, as it depends solely on the instantaneous distribution of the scalar field. This makes it a very attractive tool for studying mixing and transport properties of complicated systems such as the atmosphere. ${ }^{11,12}$ Systematic studies of this diagnostic tool have shown that areas of high effective diffusion correspond to areas of well mixed scalar and areas of low effective diffusion occur in regions of poorly mixed scalar, such as in periodic islands. ${ }^{13,14}$ For this reason we adopt the effective diffusion in this study to compare the relative sizes of the well mixed region of the time-averaged effective diffusion for a flow with periodic islands and one without islands.

The present paper is laid out as follows. In Sec. II we formulate the problem and discuss the numerical scheme used to calculate the scalar field. In Sec. III we examine the kinematic behavior of the flow. A careful study determines the parameter values for which we have periodic islands embedded in a chaotic flow and for which values we apparently have a globally chaotic flow. Section IV presents the results of the numerical simulations of the advection-diffusion equation including effective diffusion calculations and an analytical result for the TPSF problem. Our concluding remarks and some discussion are given in Sec. V.

\section{FORMULATION AND NUMERICAL SCHEME}

In an incompressible fluid flow, the evolution of a scalar tracer that has concentration $c(\mathbf{x}, t)$ is governed by the advection-diffusion equation

$$
\frac{\partial c}{\partial t}+\mathbf{u} \cdot \nabla c=\nabla \cdot(\kappa \nabla c)+S,
$$

where $\mathbf{u}$ is a prescribed incompressible velocity field, $\kappa(\mathbf{x}, t)$ is the molecular scalar diffusion, and $S(\mathbf{x}, t)$ is a source term. The current work uses the two-dimensional flow field given by Eq. (1.1) where $\omega$ is a real frequency and $x$ and $y$ are Cartesian coordinates. For simplicity we also consider the case when $\kappa(\mathbf{x}, t)$ is constant and the source term $S(\mathbf{x}, t)$ $=\cos x$, which is both steady and has zero mean.

This study examines the long time behavior of Eq. (2.1) where due to the presence of the source, the behavior of the scalar field eventually becomes periodic in time and, from a Lagrangian point of view, the source term balances molecular diffusion over one period.

Although small scales are generated in the scalar field, the specified velocity field is smooth and large scale. Consequently, the Lagrangian time scale of the scalar in this problem is much longer than the Eulerian time scale. The problem therefore lends itself to numerical solution by a semiLagrangian method, e.g., Ref. 15. We use cubic Lagrange interpolation for the scalar advection, with a Crank-Nicolson treatment of the diffusion term. This makes the scheme second-order accurate in time overall and allows much longer time steps $\Delta t$ to be used, without losing accuracy or stability, than would be possible with the Eulerian scheme used by Turner et al. ${ }^{14}$ Thus, the computation time required to reach the periodic state is greatly reduced. Nevertheless, we still find ourselves restricted in our parameter search by the computational time required to complete the runs. This is discussed further in Sec. IV.

\section{KINEMATIC PROPERTIES OF THE FLOW}

Before embarking on a full numerical simulation of Eq. (2.1), we consider the kinematic properties of the flow field [Eq. (1.1)] by examining Poincaré sections of its structure. To calculate the Poincaré sections, we first write the flow field as a system of two first order differential equations

$$
\frac{d x}{d t}=2 \sin ^{2}(\omega t) \sin y, \quad \frac{d y}{d t}=2 \cos ^{2}(\omega t) \sin x .
$$

These equations are then integrated from the set of initial conditions $\left(x_{0}, 0\right)$ and $\left(x_{0}, \pi\right)$ where

$$
x_{0}=-\pi+2 \pi(j-1) /(N-1), \quad j=1,2,3, \ldots, N,
$$

and for each initial condition the position of the fluid particle is represented by a dot in real space after each period of the flow $T=\pi / \omega$. For the resulting Poincaré sections in Fig. 1, $N=30$ and for $\omega \leq 0.5$ we integrated to $t=400 T$ and to $t$ $=200 T$ otherwise.

For large frequencies the direction of the shear flow changes rapidly and the results show that the Poincaré section has a cellular structure with four periodic "islands" centered at $(0, \pm \pi)$ and $( \pm \pi, 0)$. Between these islands is a thin band of chaos which increases in thickness as $\omega$ is reduced. This can be seen when moving from $\omega=3.5$ in Fig. 1(f) to $\omega=2$ in Fig. 1(e). As $\omega$ is decreased further the size of the islands is reduced also, because the longer flow period means that fluid particles are stretched out much further and so any island regions are eventually stretched out and removed. At $\omega \approx 0.75$ the islands disappear completely and we apparently have a globally chaotic flow. A globally chaotic flow can be seen in Fig. 1(b) where $\omega=0.5$. However, when $\omega=0.3$ we find that the globally chaotic flow changes to one with small 

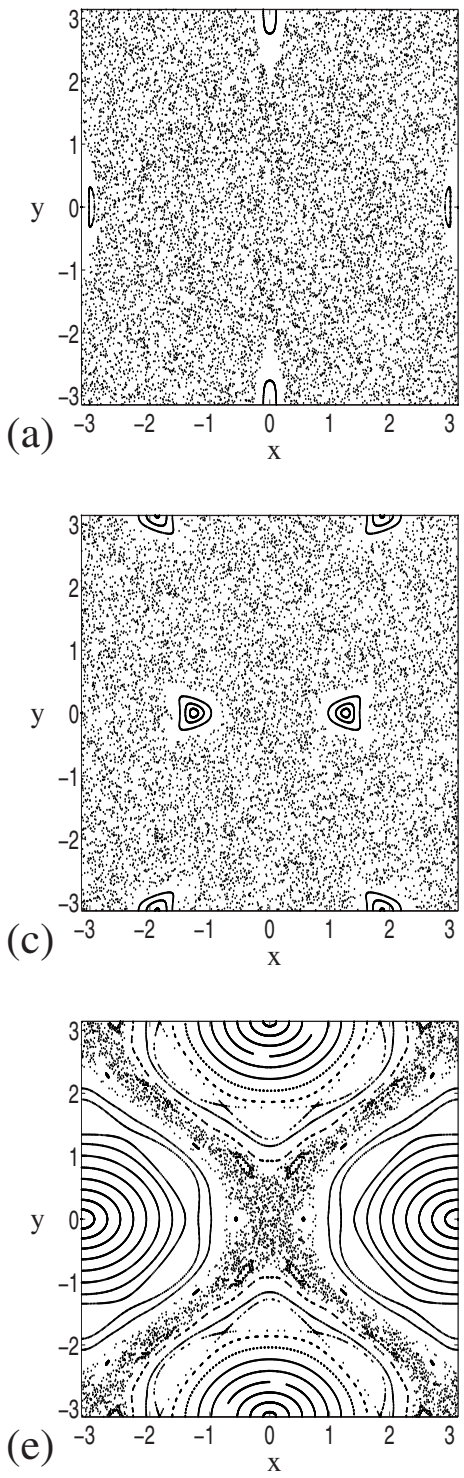
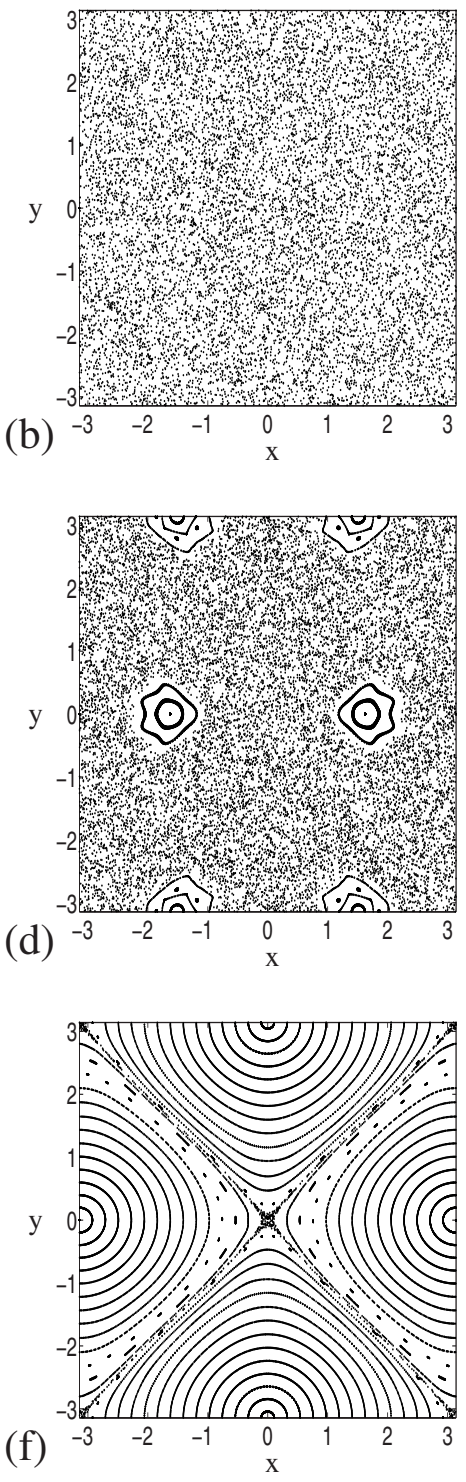

FIG. 1. Poincaré sections for the velocity field [Eq. (1.1)] for the cases (a) $\omega=0.3$, (b) $\omega=0.5$, (c) $\omega=0.8$, (d) $\omega=1$, (e) $\omega=2$, and (f) $\omega=3.5$. islands around $(0, \pm \pi)$ and $( \pm \pi, 0)$. Further reducing $\omega$ (not shown here) shows that $\omega=0.2$ is fully chaotic, but $\omega=0.1$ again has small islands. This is a resonant effect similar to that seen in the Mathieu equation. ${ }^{16}$ Thus, it is not true to say that the flow has islands for $\omega \gtrsim 0.75$; and is globally chaotic for $\omega \leqq 0.75$. Instead we have to be careful that our choice of $\omega$ generates a window where the flow does not contain islands if we wish to examine a globally chaotic flow. When we inject scalar into a globally chaotic flow we expect the scalar field to be well mixed and the maximum value of scalar $C_{\max }$ to be less than a flow case where there are islands present, as scalar builds up in such islands.

We can examine for which values of $\omega$ we expect to see the resonant islands around the points $( \pm \pi, 0)$ and $(0, \pm \pi)$ by studying a local solution about $(\pi, 0)$. Thus, inserting $x^{\prime}$ $=x-\pi$ and $y^{\prime}=y$ into Eq. (3.1) and linearizing leads to

$$
\frac{d x^{\prime}}{d t}=2 \sin ^{2}(\omega t) y^{\prime}, \quad \frac{d y^{\prime}}{d t}=-2 \cos ^{2}(\omega t) x^{\prime} .
$$

(The other three points lead to either the same system of equations, or one with the minus sign swapped over.) We now solve this system by integrating using a fourth order Runge-Kutta scheme with the initial condition $x^{\prime}=10^{-3}, y^{\prime}$ $=10^{-3}$. With these initial conditions we check the linear stability of Eq. (3.2) and we assume that linear stability implies islands in the Poincaré section and linear instability implies a globally chaotic flow. The results for the one parameter family $\omega$ are shown in Fig. 2(a), where the value 1 on the vertical axis represents chaos and 0 represents periodic orbits or islands. The results show that for $\omega \gtrsim 1.45$ there are always islands about this point, as can be seen in Figs. 1(e) and 1(f). However, as $\omega$ is reduced they disappear and then reappear at a more and more rapid rate when $\omega \leqq 0.2$. The rate at which these islands appear and disappear can be seen in Fig. 2 (b), which plots panel (a) as a function of $1 / \omega$. Here we see a regular spacing of the windows of $\omega$ values for which the islands are present. Note that for $\omega \gtrsim 0.75$ the flow can have islands at points corresponding to $(x, y) \neq( \pm \pi, 0)$ and $(0, \pm \pi)$ [cf Figs. 1(c) and 1(d)], whereas for $\omega<0.75$ they only appear to occur at these four points. It is feasible that resonant islands could occur at points other than the four mentioned above, but our exploration of the $\omega$-space consid- 

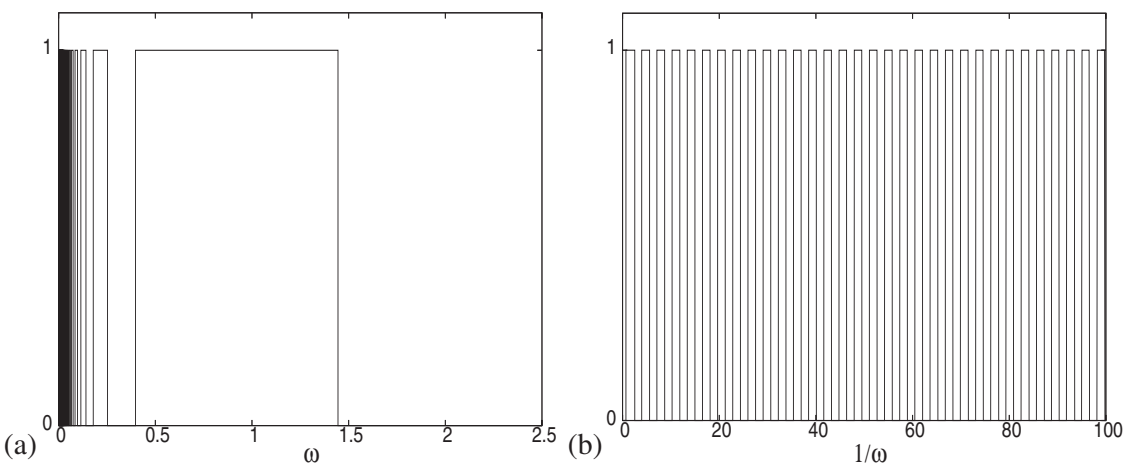

FIG. 2. Plot of (a) the parameter space for Eq. (3.2), where 0 on the vertical axis denotes periodic orbits and 1 denotes chaos. Panel (b) shows panel (a) plotted against $1 / \omega$ to show an equal spacing of windows.

ered in this paper has not revealed any such points. Thus, we assume that the four points considered here are the only such points. Now that we know when and where the periodic islands appear in the flow field we can solve the advectiondiffusion equation (2.1) and examine the evolution of the scalar field.

\section{NUMERICAL EVOLUTION OF SCALAR FIELD AND STEADY STATE ANALYSIS}

In this section we numerically study the solution of Eq. (2.1) in the large $t$ limit where the scalar field has become periodic due to the injection from the steady source. We demonstrate how the periodic scalar field and the length of various scalar contours vary over a period of the flow field, and we also show how $\overline{C_{\max }}$ varies with $\kappa$ by time averaging the maximum value of the scalar field. This section ends with an analytical approach to solve the case where Eq. (1.1) has small $\omega$. For this study we use the source distribution $S(x)$ $=\cos x$. Initially we set the scalar concentration $c(x, y, t=0)$ $=0$ and we let the system evolve with time, which gives a transient regime for early times where the scalar increases from $c=0$ up to the steady state. The length of time of this transient phase depends on both $\omega$ and $\kappa$, and increases for small $\kappa$ and large $\omega$ (short period flows). This limits the parameter range that we can study (mainly small $\kappa$ and moderate $\kappa$ with $\omega>0.8$ ), but this does not appear to restrict any results in this paper.

To analyze the scalar field in the periodic state we use the diagnostics $C_{\max }(t)$, which is the maximum value of the scalar at any given time, and the length of the contour $c=C$, which we denote by $L_{C}$. Typically we consider the contour $C=0$, as this contour always lies within the chaotic band regardless of the value of $\omega$, hence these results should be consistent. However, we do look at other scalar contour lengths later in this section.

In the periodic regime, the scalar field varies over the duration of a flow period $T=\pi / \omega$ and time averaging these diagnostics of this regime gives corresponding steady state quantities such as $\overline{C_{\max }}$ or $\overline{L_{C}}$. The variation of both $C_{\max }$ and $L_{0}$ for a selection of flow parameters is given in Fig. 3 as a function of $t / T$.

Figure 3 graphs both $C_{\max }$ and $L_{0}$ for the parameter cases $(\omega, \kappa)=\left(0.5,10^{-1}\right),(\omega, \kappa)=\left(0.5,10^{-3}\right),(\omega, \kappa)=\left(1,10^{-1}\right)$, and $(\omega, \kappa)=\left(1,10^{-3}\right)$ in panels (a), (b), (c), and (d), respectively.

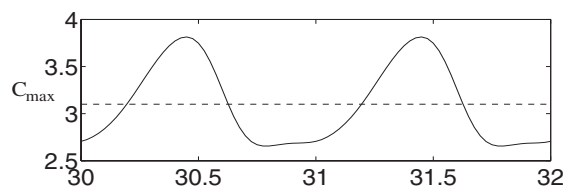

$\mathrm{L}_{0}$

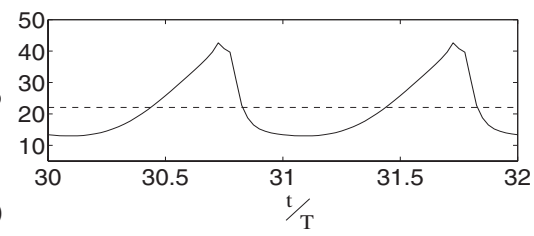

(a)
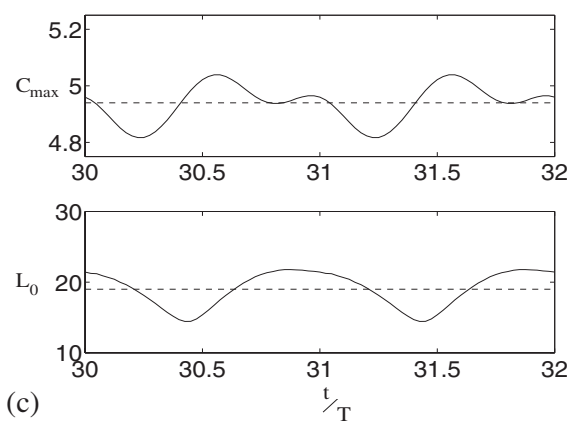
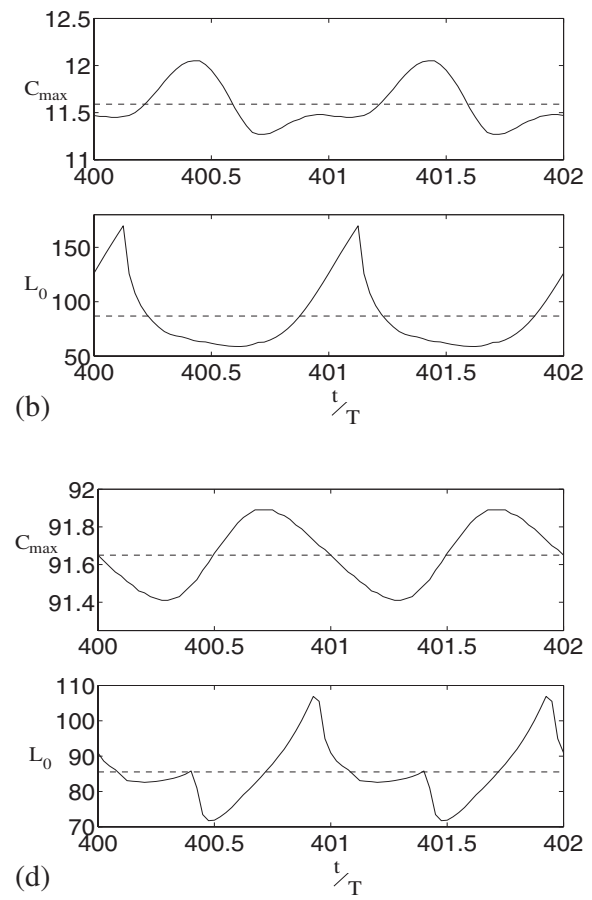

FIG. 3. Plot of both $C_{\max }$ and $L_{0}$ as a function of $t / T$ for the cases (a) $\quad(\omega, \kappa)=\left(0.5,10^{-1}\right), \quad$ (b) $\quad(\omega, \kappa)$ $=\left(0.5,10^{-3}\right)$, (c) $(\omega, \kappa)=\left(1,10^{-1}\right)$, and (d) $(\omega, \kappa)=\left(1,10^{-3}\right)$. The dashed lines in each figure represent the equivalent value of $\overline{C_{\max }}$ or $\overline{L_{0}}$. 
For each panel the respective time-averaged value of the diagnostic is given by the horizontal dotted line. We note that at the beginning and end of each period, the flow stretches the scalar approximately parallel to the $y$-axis, which is aligned with the source term, and for the middle of the flow period it is being stretched approximately parallel to the $x$-axis which shears the scalar field and allows diffusion to act strongly on the fine scale structure. Figure 3 shows that for $\omega=1$ the mean value of $C_{\max }$ is larger while the variations in $C_{\max }$ are smaller than for $\omega=0.5$. This behavior is consistent with the trapping of scalar in the islands in the $\omega=1$ case. Figure 3 also shows that the mean value of $L_{0}$ is slightly smaller while the variations in $L_{0}$ are much smaller for the $\omega=1$ case. It is not clear whether this behavior of $L_{0}$ is related to the existence or nonexistence of islands.

The general behavior of the quantities in Fig. 3 is that both $C_{\max }$ and $L_{0}$ increase to some maximum value before rapidly decreasing to a minimum value after the flow field has rotated. Note that the rotation is not instantaneous and any increase or decrease in diagnostic values is also due to the smooth rotation of the flow field. However, it appears that the actual timing of these maximum and minimum values is strongly linked to the values of $\kappa$ and $\omega$ chosen. For example, for $(\omega, \kappa)=\left(0.5,10^{-1}\right)$ in panel (a) the maximum and minimum values of $C_{\max }$ occur around $t / T=30.5$ and $t / T=31$, respectively, and for $L_{0}$ these are at $t / T=30.75$ and $t / T=31$, respectively. However, as $\omega$ is increased to 1 in panel (c) the maximum and minimum values of $C_{\max }$ now occur at $t / T=30.6$ and $t / T=30.25$ and the $L_{0}$ extremes have swapped over to $t / T=30$ and $t / T=30.5$, respectively. Similar variations can be seen by considering the $\kappa=10^{-3}$ cases. The time lag between the flow field changing and the scalar field changing is apparent in this figure, and will play a role later in determining how $\overline{C_{\max }}$ and $\overline{L_{C}}$ behave for varying $\omega$ and $\kappa$.

The results in Fig. 3 help us to understand how the scalar field behaves over the course of a time period of the flow. However, to gain more insight we consider the real space images of the scalar field which are plotted in Fig. 4. This figure shows the four cases considered in Fig. 3 plotted at the midpoint of the period $t / T=n+\frac{1}{2}$ (left panels) and the end point $t / T=n$ of the period (right panels), with red (midgray) signifying large positive values and dark blue (dark gray) signifying large negative values of scalar. In each figure the zero contour $L_{0}$ is shown as the solid black line. For $\omega$ $=0.5$ there are no islands in the flow field; this gives a scalar field with long stretched out regions of scalar. This is clearly seen for the $\kappa=10^{-3}$ in panels (c) and (d). We can also clearly see that the zero contour is much longer in this case than in the $\kappa=10^{-1}$ case [panels (a) and (b)] due to its intricate form. As we increase $\omega$ to 1 (i.e., shorten the flow period and introduce islands to the flow field) then we can see that the scalar field has a form which reflects the presence of the islands. For $\kappa=10^{-1}$ [panels (e) and (f)] four regions of scalar build up and are relatively obvious but they are much clearer in the $\kappa=10^{-3}$ result in panels $(\mathrm{g})$ and $(\mathrm{h})$. The well mixed region of scalar is larger in the latter case and in this region the scalar homogenizes producing the green region (light gray) seen in the panels. In this case one can see that the zero contour has pinched off into many smaller pieces, which allows diffusion to act and destroy these fine pieces, as seen in panels $(\mathrm{g})$ and $(\mathrm{h})$ in conjunction with Fig. 3(d). Figure 3(d) shows that $L_{0}$ decays from $t / T=400$ before growing to a small peak around $t / T=400.5$; there is then a rapid decay before rising sharply to the peak value just before $t / T=401$. This is the shear flow breaking off the $C=0$ contour into smaller pieces which are then stretched at first to give the increase in $L_{0}$. However, they then become "too" stretched and of a fine scale allowing diffusion to destroy these elements and giving the rapid drop in $L_{0}$.

In Fig. 4 it is clear that as $\kappa$ is reduced $L_{0}$ increases, but what is not so clear is how does the existence, or lack thereof, of islands affect the length of these contours and their dependence on $\kappa$ ? This question is addressed in Fig. 5 which plots the time-averaged contour length for $C=0,5$, and 20 for both $\omega=0.2$ and 1 as a function of $\kappa$. For the flow with no islands $(\omega=0.2)$ in panel (a) we see that each of the three contour values has a length which grows almost as $\kappa^{-1 / 2}$, represented by line 4 ; this is commonly observed in chaotic flows. ${ }^{17}$ The $C=20$ contour line (line 3 ) grows most like $\kappa^{-1 / 2}$ while the $C=0$ and 5 contour lines grow at a slightly slower rate. The $\overline{L_{20}}$ result initially grows at a different rate to the other two because when the $C=20$ contour first exists for $\kappa=10^{-2}$, this contour is in a region of low scalar stretching where the larger values of scalar are observed, and it is not until $\kappa=0.003$, where $\overline{C_{\max }}$ has increased, that it is in a region of the flow where the contours grow more like $\kappa^{-1 / 2}$. A similar situation occurs for a flow with islands $(\omega=1)$ with the only apparent difference between this panel and that for $\omega=0.2$ is now all three contours grow as $\kappa^{-1 / 2}$ for small $\kappa$. This shows that in a flow without islands, the time-averaged contour stretching is reduced in the middle of the chaotic band seen in Figs. 4(c) and 4(d). However, this subtle change in growth rate is not expected to be important in determining how much scalar builds up in the islands, as many other factors are significant too.

In Fig. 6 we plot $\overline{C_{\max }}(\kappa)$ for various values of $\omega$ on a $\log -\log$ scale. In panel (a) we consider small values of $\omega$ $=0.01,0.1,0.2,0.3,0.5$, and 0.8 and these are labeled 1, 2, 3, 4,5 , and 6 , respectively, and in panel (b) we consider larger values of $\omega=0.5,0.8,0.9,1.0,1.2,2.0$, and 3.5 which are labeled $1,2,3,4,5,6$, and 7 , respectively. The intermediate values of $\omega=0.5$ and 0.8 are plotted on both panels to link the two together and give the reader a sense of the whole parameter range without a congested figure. For the small values of $\omega$ in panel (a) we see a typical behavior emerging. When $\omega$ is moderately small and the flow field contains no islands, such as for lines 3 and 5 , we see that $\overline{C_{\max }}$ does not grow as an inverse power of $\kappa$, and in fact when these two results are plotted on a log-linear plot in Fig. 7, they give approximate straight lines which suggests a growth of $\overline{C_{\max }}$ $\propto-\ln \kappa$. The reason for this growth dependence is not clear, and it is not true of all globally chaotic flows. We know this is the case because as we reduce $\omega$ further to 0.1 and 0.01 , both of which have no resonance islands from Sec. III, we find that $\overline{C_{\max }}$ begins to grow approximately as an inverse power of $\kappa$. [This can be seen by considering Fig. 2(b).] In fact the $\omega=0.01$ result (line 1) can be shown to grow like $\kappa^{-1}$ plus some smaller correction terms. This analysis is carried 
(a)

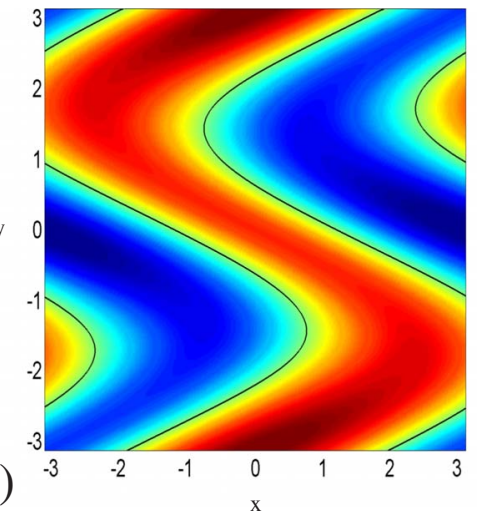

(c)

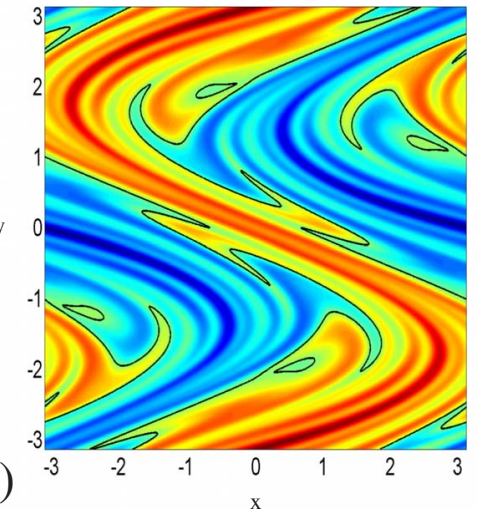

(e)

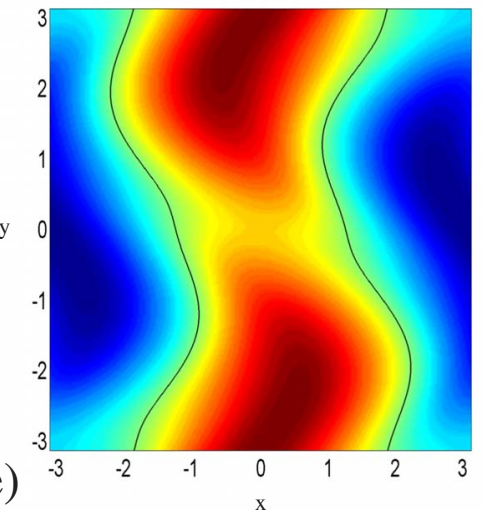

(g)

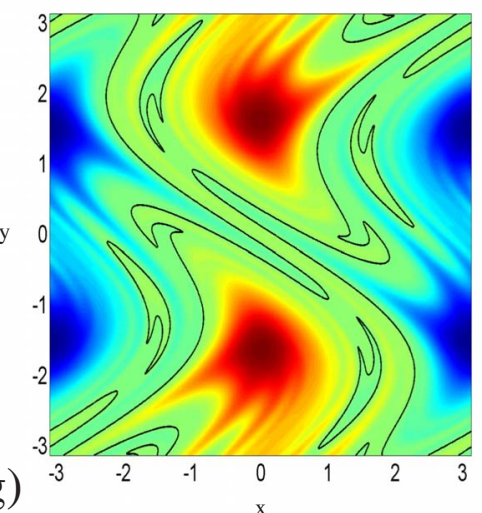

(b)

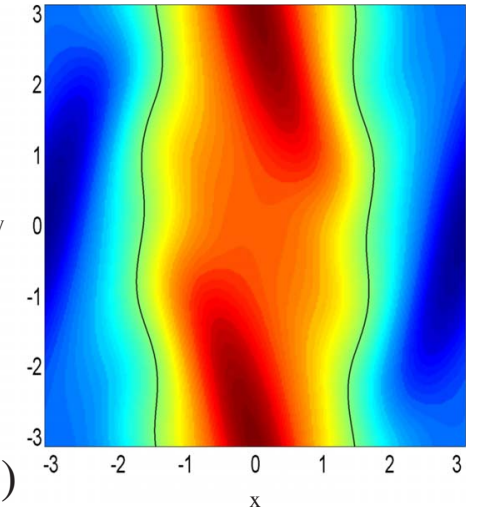

(d)

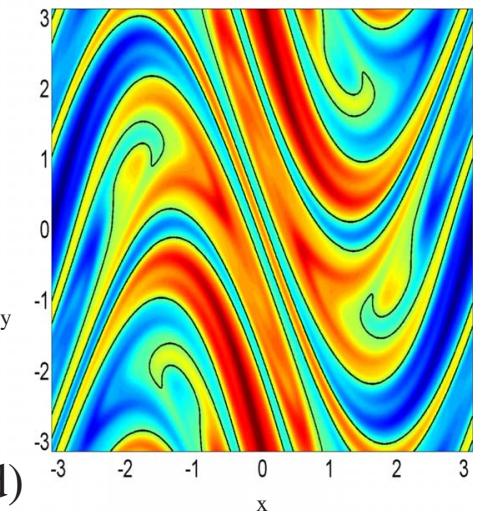

(f)
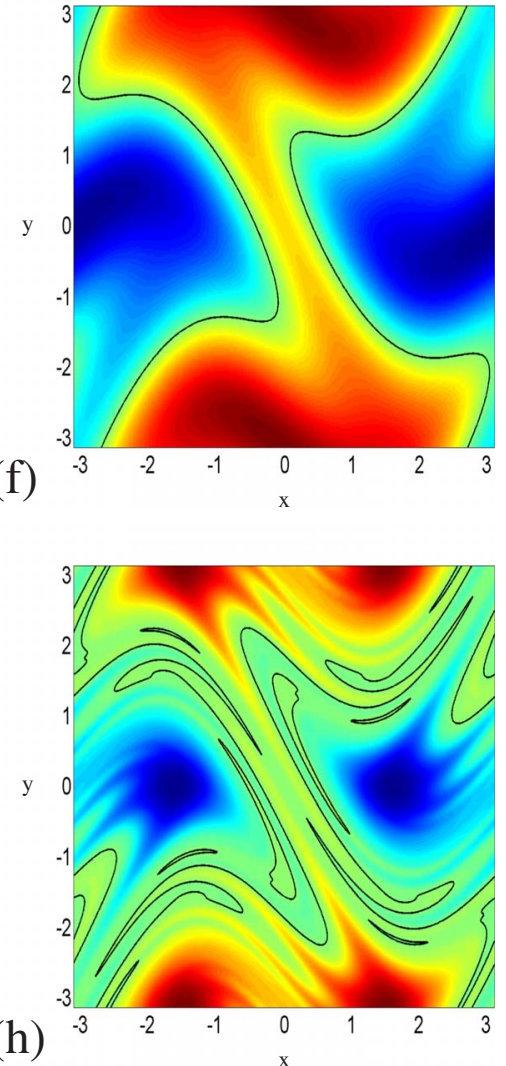

FIG. 4. (Color online) Plots of the scalar field $c(x, y, t)$ in the periodically steady state at the midpoint of a period (left panels) and the end of a period (right panels) for [(a) and (b)] $(\omega, \kappa)$ $=\left(0.5,10^{-1}\right), \quad[(\mathrm{c})$ and $(\mathrm{d})](\omega, \kappa)$ $=\left(0.5,10^{-3}\right), \quad[(\mathrm{e})$ and $(\mathrm{f})] \quad(\omega, \kappa)$ $=\left(1,10^{-1}\right)$, and $[(\mathrm{g})$ and $(\mathrm{h})](\omega, \kappa)$ $=\left(1,10^{-3}\right)$. Red (midgray) corresponds to large positive values of $c$ and dark blue (dark gray) corresponds to large negative $c$. The black line is the contour $c=0$. out in Sec. IV A. For the cases in panel (a) where there are islands, $\omega=0.3$ (line 4) and $\omega=0.8$ (line 6), we see that for larger $\kappa$ these results grow much like their nonisland counterparts, down to some critical value of $\kappa$ where the effect of the islands becomes significant, and then they begin to grow as $\kappa^{-\alpha}$ for some $\alpha \in(0,1]$.

An interesting comparison can be drawn with the work of Haynes and Vanneste ${ }^{18}$ who found that the decay rate of 


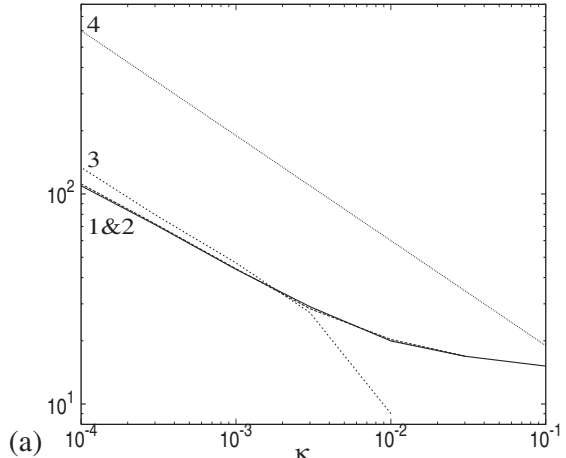

(b)

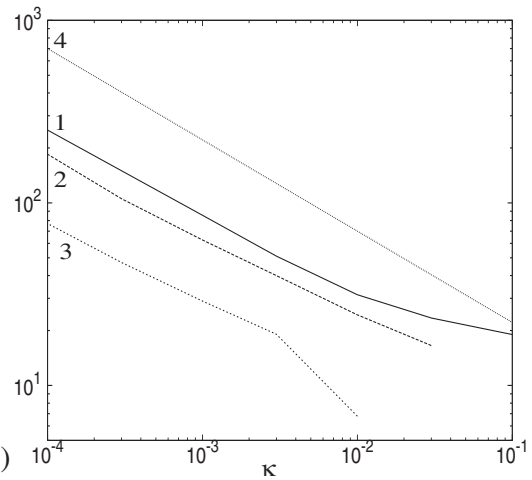

FIG. 5. Plot of $\overline{L_{C}}(\kappa)$ for (a) $\omega=0.2$ and (b) $\omega=1$ where the contours $C$ $=0,5$, and 20 are labeled $1-3$ and line 4 is $\propto \kappa^{-1 / 2}$. passive scalar variance (with no source) has a logarithmic dependence on $\kappa$ as $\kappa \rightarrow 0$ in the case when transport is "locally controlled" that is by the spectrum of Lyapunov exponents. This slow dependence, for random flows without islands, may be connected with the slow, logarithmic growth in $\overline{C_{\max }}$ as $\kappa \rightarrow 0$ that we observe in flows without islands. ${ }^{19}$ On the other hand the case of large-scale "globally controlled" modes in Ref. 18 leads to a power law dependence of the decay rate on $\kappa$. In this case the large-scale scalar field can be thought of as giving a source of scalar fluctuations to smaller scales, and is similar to the way in which islands in our flows trap and slowly release scalar, giving a power law behavior for $\overline{C_{\max }}$. Possible connections between the scaling laws for forced and freely decaying scalars merit further investigation.

In the corresponding large $\omega$ figure, Fig. 6(b), we observe that as $\omega$ increases, the value of the critical $\kappa$, where the role of the islands becomes significant, moves to larger $\kappa$ values until by $\omega=1$ (line 4 ), values of $\overline{C_{\max }}$ appear to grow $\propto \kappa^{-\alpha}$ over the whole range of $\kappa$ considered. The corresponding value of $\alpha$ for this growth itself increases with $\omega$ until $\omega \approx 3.5$ (line 7 ) where it has the approximate value 1 . This is because the scalar builds up in the islands and gives a scalar distribution of $c(x, y) \approx 1 /(2 \kappa)(\cos x-\cos y)$ as noted in Ref. 14. The results for $\omega=2.0$ and 3.5 (lines 6 and 7) are not computed all the way down to $\kappa=10^{-4}$ as the other results are because as $\omega$ becomes larger, the number of flow periods that have to be completed before a steady state is achieved increases greatly; however, results down to this value of $\kappa$ are not required to get a clear picture of how the scalar behaves for these values of $\omega$. One question still remains: can we link the value of the exponent $\alpha$ to the size of the periodic islands?

In Fig. 8 we plot the scaling exponent of $\overline{C_{\max }}, \alpha$, as a function of the area of the periodic islands in the flow field. For $\omega$ values between 1.2 and 3.5, we calculate the area of the islands by integrating Eq. (3.1) from a regularly spaced array of positions in the domain $x \in[0, \pi], y \in[0, \pi]$ and observing whether or not the particle path completes a periodic cycle, i.e., it comes back to its starting position. The area of the islands is then calculated as the fraction of periodic orbits compared to the chaotic orbits scaled up to the whole $[-\pi, \pi]^{2}$ domain. The corresponding value of $\alpha$ on the other hand is calculated by taking the gradient between the values $\kappa=10^{-3}$ and $3 \times 10^{-3}$ in Fig. 6(b). Both these calculations are prone to numerical error; however, test calculations have shown that the maximum error for both values is around $3 \%$. Thus, Fig. 8 shows that by investigating this region of parameter space, we find a correlation between the size of the islands and the scaling exponent $\alpha$. It is not clear if the correlation could be made linear by plotting $\alpha$ against another measure of the islands such as their circumference because although the islands are clearly defined in the flow field, they are surrounded by regions of weak stretching which could also be significant to the build up of scalar within the islands. Thus, the calculation of a circumference is difficult. Also, if we consider Figs. 1(d) and 4(h), we see that the region of scalar build up in Fig. 4(h) is larger than the physical islands in Fig. 1(d). The scalar field also has fingers of scalar being stretched out which may be significant in the calculation of $\alpha$. If this investigation were continued to smaller island areas then we may also find another branch of

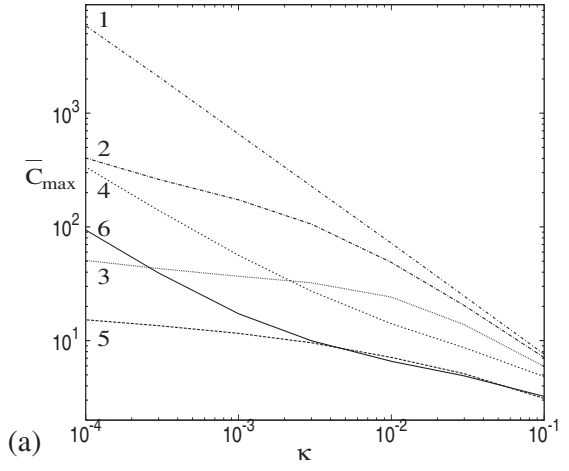

(b)

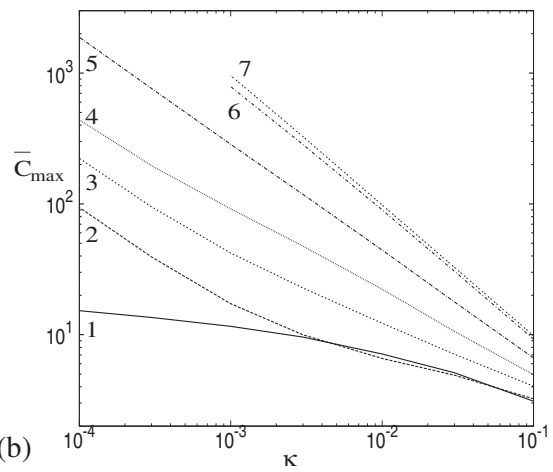

FIG. 6. Plot on a $\log -\log$ scale of $\overline{C_{\max }}(\kappa)$ for (a) $\omega=0.01,0.1,0.2,0.3$, 0.5 , and 0.8 labeled $1,2,3,4,5$, and 6 , respectively, and (b) $\omega=0.5,0.8,0.9$, 1.0, 1.2, 2.0, and 3.5 labeled 1, 2, 3, 4, 5,6 , and 7 , respectively. Panel (a) is the corresponding small $\omega$ figure and panel (b) is the large $\omega$ figure, with the results $\omega=0.5$ and 0.8 appearing on both for continuity. 


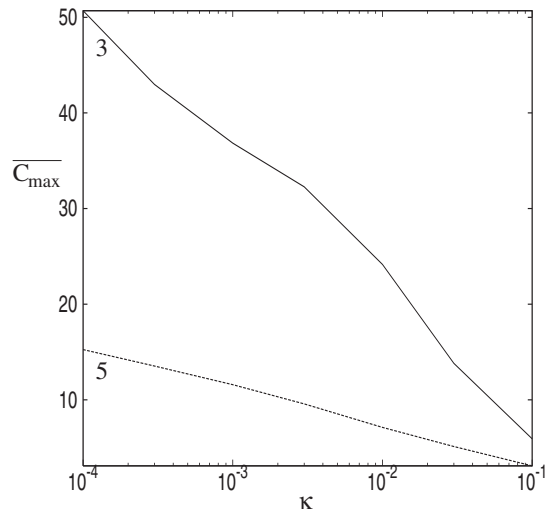

FIG. 7. Plot of $\overline{C_{\max }}(\kappa)$ on a log-linear plot for $\omega=0.2$ and 0.5 marked as 3 and 5 , respectively.

solutions appearing in Fig. 8. If this were to happen it would show that $\alpha$ does not correlate exactly with the area of the islands, but it could help to understand how $\alpha$ scales.

From Fig. 6(b) we see that $\overline{C_{\max }}$ grows like $\kappa^{-\alpha}$ in the large $\omega$ limit and moreover that there is a correlation between $\alpha$ and the size of the flow islands. However, the exact scaling exponent for small $\omega$ is less clear, and this limit is examined further in the next section by considering a simplified model of the flow field.

\section{A. Understanding the small $\omega$ limit: Analysis of the TPSF}

To understand the scalar distribution in the small $\omega$ limit of the shear flow [Eq. (1.1)], we simplify the problem to one that can be analyzed more easily. We consider the flow consisting of two shearing motions where we "switch" from one to the other after a given time $t_{s}$; this is known in literature as the TPSF. ${ }^{4,5}$ Thus, for one period our flow becomes

$$
\mathbf{u}= \begin{cases}2 \sin x \hat{\mathbf{y}}, & 0<t \leq t_{s}, \\ 2 \sin y \hat{\mathbf{x}}, & t_{s}<t \leq 2 t_{s} .\end{cases}
$$

This simplified flow takes away the complication of the flow axis varying continuously between these two shear flows as in Eq. (1.1). The analysis in this section shows that the solution for the scalar field in the initial half of the period is

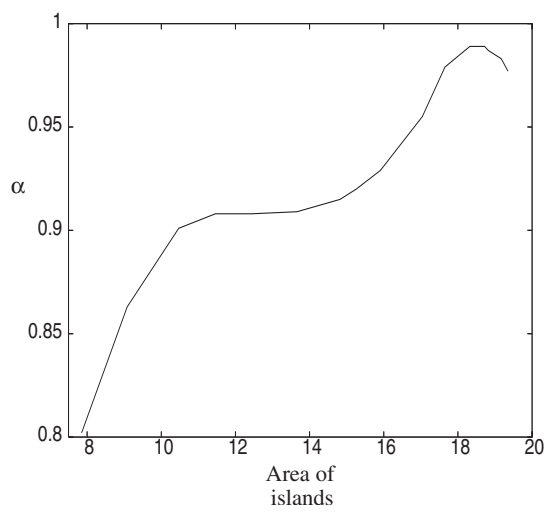

FIG. 8. Plot of the scaling exponent of $\overline{C_{\max }}, \alpha$, as a function of the area of the periodic islands in the flow field. easy to examine analytically, while the second half leads to a boundary layer type problem for small $\kappa$. Before carrying out an analytical study we first examine the numerical results.

Figure 9(a) plots $C_{\max }(t)$ for the TPSF with $t_{s}=2500$ for $\kappa=10^{-1}, 10^{-2}, 10^{-3}$, and $10^{-4}$ labeled $1,2,3$, and 4 , respectively. We observe that over the first half of the period, $C_{\max }$ increases to $\kappa^{-1}$, if $t_{s}$ is large enough to allow this. This happens because for this time the shear flow acts parallel to the injection of scalar from the source and scalar builds up over this half period until it balances diffusion and $c(x, y)$ $=\kappa^{-1} \cos x$. However, for $t_{s}<t<2 t_{s}$ the shear flow acts perpendicular to the source, effectively stretching out the scalar into very fine filaments, which diffusion can then act on and remove. Thus, at some point $C_{\max }$ reaches a constant value, a steady state; however, for smaller values of $\kappa$ the diffusion is weaker and it takes longer for the tracer to cascade to the scales at which diffusion balances stretching. Figure 9(b) again plots $C_{\max }$ in this TPSF for $\kappa=10^{-3}$, but with two values of $t_{s}$. The outcome is that the steady state for $t_{s}<t$ $<2 t_{s}$ is independent of the form of $c$ at $t=t_{s}$, hence we can analyze this part of the period by considering the steady form of Eq. (2.1) for simplicity.

By examining the real space plot of the scalar field $c(x, y)$ in the steady state for $t_{s}<t<2 t_{s}$ in Fig. 10, we can clearly see that as $\kappa$ is reduced [see panel (b) for $\kappa=10^{-3}$ ] the scalar distribution becomes concentrated around $y=0$ and $\pm \pi$. This is not as clear for larger values of $\kappa$, such as $\kappa$ $=10^{-1}$ in panel (a), and so to understand how $C_{\max }$ varies with $\kappa$ we investigate the limit of small $\kappa$.

\section{Steady solution for $t \leq t_{s}$}

For the first half of the flow period, the steady form of Eq. (2.1) simplifies to

$$
2 \sin x \frac{\partial c}{\partial y}=\kappa \nabla^{2} c+\cos x
$$

This equation can easily be solved by seeking a solution for $c(x, y)$ in terms of its Fourier components

$$
c=c_{1,0} e^{i x}+c_{1,1} e^{i(x+y)}+c_{1,-1} e^{i(x-y)}+c_{0,1} e^{i y}+\cdots+\text { c.c. },
$$

and then equating the coefficients of each component. Doing this we find that $c_{1,0}=1 /(2 \kappa)$ and all other coefficients are zero. Thus,

$$
c=\frac{e^{i x}}{2 \kappa}+\text { c.c. }=\frac{\cos x}{\kappa},
$$

and hence $C_{\max }=\kappa^{-1}$ for large times, as observed in Fig. 9(a).

\section{Steady solution for $t_{s}<t<2 t_{s}$}

For the second half of the flow period the steady form of Eq. (2.1) takes the similar form

$$
2 \sin y \frac{\partial c}{\partial x}=\kappa \nabla^{2} c+\cos x
$$

In this case, as the flow is independent of $x$ we can assume that the scalar is harmonic in $x$ with wavenumber 1 . Thus, for small $\kappa$ we are seeking a solution to Eq. (4.1) in the form 


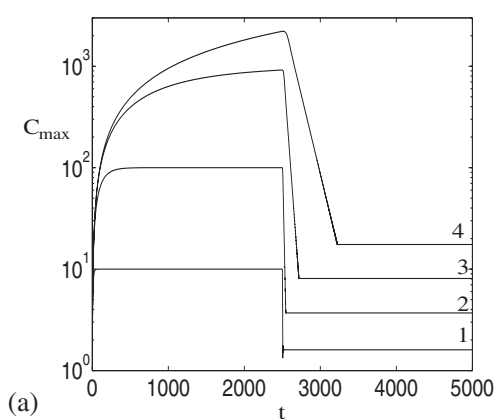

(a)

$$
c=\left[c_{0}(y, t)+\kappa c_{1}(y, t)+O\left(\kappa^{2}\right)\right] e^{i x}+\text { c.c. },
$$

where we denote $\tilde{c}=c_{0}(y, t)+\kappa c_{1}(y, t)+O\left(\kappa^{2}\right)$. Substituting this into Eq. (4.1) gives at $O(1)$,

$$
2 i c_{0} \sin y=\frac{1}{2},
$$

and at $O(\kappa)$,

$$
2 i c_{1} \sin y=\left(\frac{\partial^{2}}{\partial y^{2}}-1\right) c_{0} .
$$

Solving these two algebraic equations for $c_{0}$ and $c_{1}$ gives

$$
c=\left(-\frac{i}{4 \sin y}-\frac{\kappa \cos ^{2} y}{2 \sin ^{4} y}\right) e^{i x}+\text { c.c. }
$$

However, this expression has a singularity as $y \rightarrow 0$; in fact in this limit the above expression becomes

$$
c=\left(-\frac{i}{4 y}-\frac{\kappa}{2 y^{4}}\right) e^{i x}+\text { c.c. },
$$

which contradicts Fig. 9 that shows a finite value of $C_{\max }$ at $y=0$. Thus, using this knowledge and Fig. 10(b), we know that there is a layer about $y=0$ (or similarly $y= \pm \pi$ ) where the diffusive effects are more important. The above result can also be found in Ref. 20; however, the inner solution is only calculated numerically while below we give the analytical form of this solution.

Substituting Eq. (4.3) into Eq. (4.2) and expanding about $y=0$ leads to the equation

$$
\frac{\partial^{2} \tilde{c}}{\partial y^{2}}-\left(\frac{2 i y}{\kappa}+\kappa\right) \tilde{c}=-\frac{1}{2 \kappa} .
$$

We solve this problem by calculating the Green's function $G(y, s)$ which satisfies

$$
\frac{\partial^{2} G}{\partial y^{2}}-\left(\frac{2 i y}{\kappa}+\kappa\right) G=\delta(y-s),
$$

where $\delta(X)$ is the Dirac delta function. The Green's function must also satisfy the jump conditions

$$
[G]_{y=s^{-}}^{y=s^{+}}=0 \text { and } \quad\left[\frac{\partial G}{\partial y}\right]_{y=s^{-}}^{y=s^{+}}=1 .
$$

The Green's function is calculated by transforming the homogeneous form of Eq. (4.7) to the Airy equation

$$
\frac{\partial^{2} G}{\partial Y^{2}}-Y G=0
$$

by changing variables to $Y=A y$, where $A^{3}=2 i / \kappa$, and neglecting the small $\kappa$ term in the brackets. To have exponential decay for large $Y$ we choose the solution

$$
G(Y, s)=\alpha(s) A i(Y) .
$$

We convert this expression back into the $y$-space and we take $A=(2 / \kappa)^{1 / 3} e^{i \pi / 6}$ for $s<y$ and $A=(2 / \kappa)^{1 / 3} e^{5 i \pi / 6}$ for $y<s$. (The third root gives $y$ purely imaginary and is of no significance for this problem.) This gives the Green's function as

$$
G= \begin{cases}\alpha(s) A i(f y), & s<y, \\ \beta(s) A i\left(e^{2 \pi i / 3} f y\right), & y<s,\end{cases}
$$

where $f=(2 / \kappa)^{1 / 3} e^{i \pi / 6}$ and $\alpha(s)$ and $\beta(s)$ are arbitrary functions. Solving for $\alpha(s)$ and $\beta(s)$ using the jump conditions gives

$$
G= \begin{cases}-2 \pi e^{i \pi / 6} f^{-1} A i\left(e^{2 \pi i / 3} f s\right) A i(f y), & s<y, \\ -2 \pi e^{i \pi / 6} f^{-1} A i(f s) A i\left(e^{2 \pi i / 3} f y\right), & y<s .\end{cases}
$$

Thus, the general solution for $\widetilde{c}(y)$ is

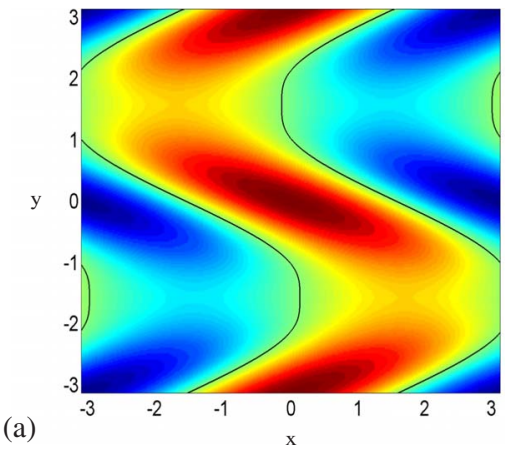

(b)

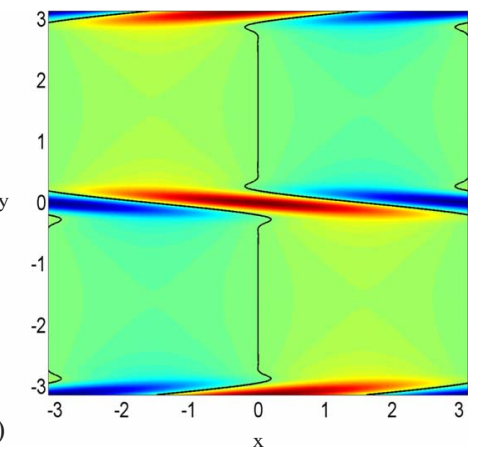

FIG. 10. (Color online) Plot of the scalar field for the TPSF for $t_{s}<t$ $<2 t_{s}$ and for (a) $\kappa=10^{-1}$ and (b) $\kappa$ $=10^{-3}$. Red (midgray) corresponds to large positive values of $c$ and dark blue (dark gray) corresponds to large negative $c$. The black line is the contour $c=0$. 


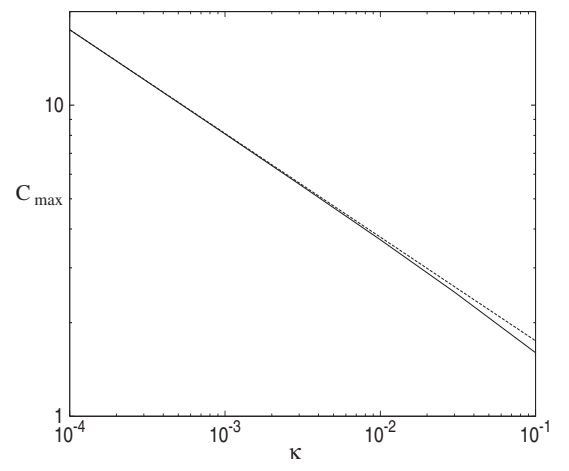

FIG. 11. Plot of $C_{\max }(\kappa)$ for the TPSF $\left(t_{s}<t<2 t_{s}\right)$, with the simulation results as the solid line and the asymptotic result [Eq. (4.12)] as the dashed line.

$$
\widetilde{c}(y)=-\int_{-\infty}^{\infty} \frac{G(s, y)}{2 \kappa} d s,
$$

which when integrated gives

$$
\begin{aligned}
\tilde{c}= & \frac{\pi}{\left(2 \kappa^{2}\right)^{1 / 3}}\left[\frac{e^{i \pi / 6} \kappa^{1 / 3}}{3 \times 2^{1 / 3}} A i(f y)+A i(f y) \int_{0}^{y} A i\left(e^{2 \pi i / 3} f s\right) d s\right. \\
& \left.+A i\left(e^{2 \pi i / 3} f y\right) \int_{y}^{\infty} A i(f s) d s\right] .
\end{aligned}
$$

By using the asymptotic expressions from pp. 448 and 449 of Ref. 21, the leading order term of the large $y$ expansion of Eq. (4.11) can be shown to be

$$
\tilde{c} \sim-\frac{i}{4 y},
$$

which agrees with Eq. (4.6). Also, from the numerical simulations in this section we know that the maximum value of $c$ occurs at $y=0$, so evaluating Eq. (4.11) at $y=0$ gives

$$
c=2 \widetilde{c} \cos x, \quad \widetilde{c}=\frac{\pi A i(0)}{3^{1 / 2} 2^{2 / 3} \kappa^{1 / 3}},
$$

and

$$
C_{\max } \sim 0.811 \kappa^{-1 / 3} .
$$

This asymptotic value of $C_{\max }$ (dashed line) is plotted with the value obtained from the full numerical simulation (solid line) in Fig. 11. Note that the agreement is excellent for small $\kappa$.

Linking this result back to the $\omega=0.01$ result (line 1 ) of Fig. 6(a) shows that as $\omega \rightarrow 0$ then $\overline{C_{\max }}=\frac{1}{2}\left[\kappa^{-1}+0.811 \kappa^{-1 / 3}\right.$ $\left.+o\left(\kappa^{-1 / 3}\right)\right]$, which when compared to the $\omega=0.01$ result shows reasonable agreement. The agreement would improve as $\omega$ is reduced further, but gaining this result numerically is beyond the parameter range that we can easily compute.

\section{B. Effective diffusion}

A useful diagnostic for examining scalar fields is the effective diffusion of the scalar field. ${ }^{9,10,13,14}$ This diagnostic has the useful property that it is large in regions where the scalar field is well mixed, and low in regions of poorly mixed scalar, ${ }^{9,13}$ thus in this section we shall apply it to scalar fields from flows with and without islands to try to understand how the scalar mixes within these flows.

The time evolution of the scalar in the transformed isotracer coordinates is determined as a function of area in two dimensions ${ }^{9}$ or volume in three dimensions. ${ }^{10}$ For the two-dimensional case we define the area of a contour $C$ as the region bounded by this contour, i.e., everywhere where $c(\mathbf{x}, t) \leq C$. Thus, we write

$$
A=\iint H[C-c(\mathbf{x}, t)] d A,
$$

where $H(X)$ is the Heaviside function. Under this change of coordinates Eq. (2.1) becomes

$$
\frac{\partial C}{\partial t}=\frac{\partial}{\partial A}\left(K_{\mathrm{eff}}(A, t) \frac{\partial C}{\partial A}\right)+\langle S\rangle_{A},
$$

where the effective diffusion can be written as

$$
K_{\mathrm{eff}}=\frac{\partial A}{\partial C} \int_{\gamma(C, t)} \kappa|\nabla c| d s=\frac{\left\langle\kappa|\nabla c|^{2}\right\rangle_{A}}{(\partial C / \partial A)^{2}} .
$$

The contour $\gamma(C, t)$ bounds the region $A$ at a time $t$, and the weighted contour average of a quantity $\chi$ is given by

$$
\langle\chi\rangle_{A}=\frac{\partial}{\partial A} \int_{c \leq C(A, t)} \chi d A=\left(\int_{\gamma} \frac{d s}{|\nabla c|}\right)^{-1} \int_{\gamma} \chi \frac{d s}{|\nabla c|} .
$$

In this study we calculate the effective diffusion of a particular scalar contour $C$ using both forms of Eq. (4.15) and this then acts as a check on the resolution of our code. For more information on numerical evaluation by the second method in Eq. (4.15), see Refs. 13 and 14.

In this section we consider the time-averaged form of $K_{\text {eff }}$ to give a view of how the scalar is mixed over one period of the flow. Thus, we define the time-averaged effective diffusion as

$$
\hat{K}_{\text {eff }}=\overline{K_{\text {eff }} \frac{\partial C}{\partial A}} / \overline{\frac{\partial C}{\partial A}} .
$$

Previous studies have shown that areas of large effective diffusion correspond to regions of the flow where the scalar is well mixed. ${ }^{9,13,14}$ Thus, we consider how the timeaveraged effective diffusion may look for two flow fields, one without islands $(\omega=0.5)$ and one with islands $(\omega=1)$. These effective diffusivities $\hat{K}_{\text {eff }}$ are plotted for $\omega=0.5$ in Fig. 12(a) and $\omega=1$ for Fig. 12(b) both for $\kappa=10^{-1}, 10^{-2}, 10^{-3}$, and $10^{-4}$ labeled $1,2,3$, and 4, respectively. In Fig. 12 we plot $\hat{K}_{\text {eff }}$ as a function of $A$, thus $A=0$ corresponds to $C=-C_{\max }, A=4 \pi^{2}$ corresponds to $C=C_{\max }$ and $A=2 \pi^{2}$ at $C=0$.

One clear distinction to note from the two panels in Fig. 12 is that $\hat{K}_{\text {eff }}(A)$ for $\omega=0.5$ has a much wider profile than for $\omega=1$, i.e., that $\hat{K}_{\text {eff }}>\frac{1}{2} \max \left(\hat{K}_{\text {eff }}\right)$ over a much larger range of area values for $\omega=0.5$. For $\kappa>10^{-2}, \hat{K}_{\text {eff }}$ is larger for most values of $A$ for $\omega=0.5$, showing that a globally chaotic flow mixes scalar better than a flow with islands $(\omega=1)$. However, as $\kappa$ is reduced to $10^{-4}$ (line 4 in Fig. 12) we see that al- 

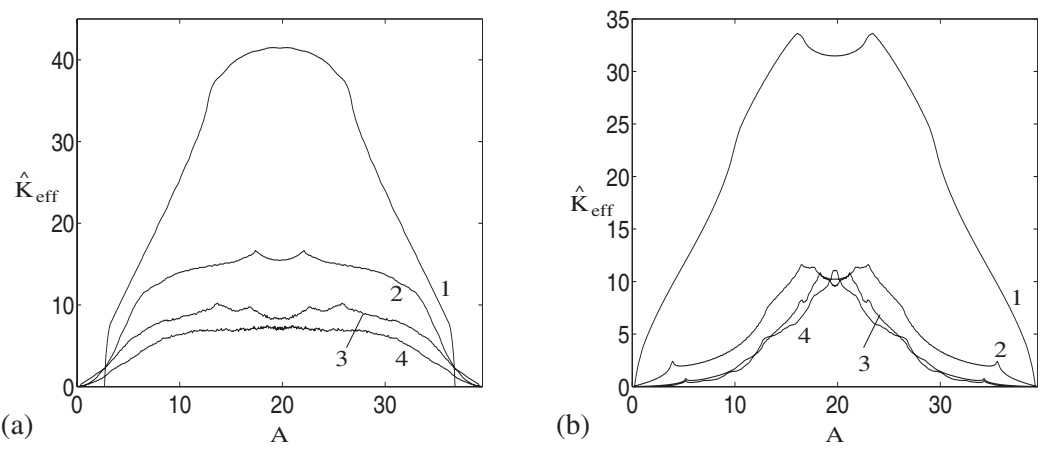

FIG. 12. Plot of the time-averaged effective diffusion $\hat{K}_{\text {eff }}(A)$ for (a) $\omega=0.5$ and (b) $\omega=1$ with curves for $\kappa$ $=10^{-1}, 10^{-2}, 10^{-3}$, and $10^{-4}$ labeled $1,2,3$, and 4 , respectively.

though $\hat{K}_{\text {eff }}$ has a larger value (suggesting that the scalar is generally better mixed) over most of the domain for $\omega=0.5$, there is a region around $A=2 \pi^{2}$ for $\omega=1$ where $\hat{K}_{\text {eff }}$ is larger, suggesting that the chaotic band seen in Figs. 4(g) and 4(h) contains scalar which is better mixed than the scalar seen in Figs. 4(c) and 4(d). Another point to note is that for $\omega=0.5$, there is a clear difference between the $\kappa=10^{-3}$ (line 3) and the $\kappa=10^{-4}$ (line 4) results, while for $\omega=1$ these two results are much more similar. This suggests that if $\hat{K}_{\text {eff }}$ has a finite limit as $\kappa \rightarrow 0$ then the presence of islands may cause this limit to be obtained for a larger value of $\kappa$.

Turner et al. ${ }^{14}$ calculated results for $\hat{K}_{\text {eff }}$ for $\kappa=10^{-2}$, $10^{-3}$, and $10^{-4}$ using an Eulerian code. The restrictions using this code mean that steady states were hard to reach as they require very long time integrations, and in fact the results for $\kappa=10^{-3}$ and $10^{-4}$ (along with $10^{-5}$ and $10^{-6}$ ) in Fig. 13 of Turner et al. ${ }^{14}$ are not at a steady state, as speculated in the previous paper. However, the semi-Lagrangian code in the current paper allows us to integrate for long time periods, so we can be sure that the results in Fig. 12(b) really are steady. Thus, for these steady results we note that $\hat{K}_{\text {eff }}$ for $10^{-3}$ and $10^{-4}$ are fairly similar; however, we would still need to examine much smaller values of $\kappa$ to fully determine whether or not there is a finite limit for $\hat{K}_{\text {eff }}$ as $\kappa \rightarrow 0$, and this question is left for future research.

\section{CONCLUSIONS AND DISCUSSION}

In this paper we studied the behavior of a scalar field in a velocity field with a steady source of scalar. The velocity field takes the form of a shear flow which changes from being approximately parallel to the $y$-axis to being approximately parallel to the $x$-axis periodically with period $T$ $=\pi / \omega$. The parameter $\omega$ can be varied and the flow field changes from configurations where the Poincaré section is globally chaotic to situations where it has islands surrounded by a chaotic sea. As $\omega$ is decreased the globally chaotic and island cases alternate at the rate $1 / \omega$ due to a resonance effect of the flow field. Throughout this work we have concentrated on the large time form of the scalar when it is in a periodic state and we can sensibly define a time average for the scalar diagnostics over each period of the flow.

The results show that the time-averaged lengths of scalar contours in the chaotic region approximately increase as $\kappa^{-1 / 2}$ for both flows with islands and without; however, the time-averaged maximum value of the scalar, $\overline{C_{\max }}$, depends more strongly on the size of the flow islands with larger islands giving a larger value of $\overline{C_{\max }}$. The flow field in Eq. (1.1) is not typical of an atmospheric flow; however, this result is still of interest to those investigating the build up of pollutants in the atmosphere or other fluid flows. For large values of $\omega$, the scalar field and flow field have an almost cellular structure, and $\overline{C_{\max }} \sim \kappa^{-1}$, while as $\omega$ is decreased $\overline{C_{\max }} \sim \kappa^{-\alpha}$, where $\alpha$ decreases from 1 and there is a correlation between $\alpha$ and the area of the flow islands. This algebraic growth, as opposed to the $-\ln \kappa$-type growth seen in the simulations, also begins to occur for smaller values of $\kappa$ as $\omega$ is reduced. At $\omega \approx 0.75$ the periodic islands disappear and at this point $\overline{C_{\max }}$ appears to increase like $-\ln \kappa$. Thus, the presence of the islands in the flow affects the scalar field by inhibiting mixing and allowing scalar concentrations to build up.

For small values of $\omega$ where the flow field exhibits a resonance effect, $\overline{C_{\max }}$ again appears to grow like $\kappa^{-\alpha}$ for an exponent $\alpha \in(0,1]$, but on careful examination it actually grows as $\kappa^{-\alpha}$ plus a small correction term. For the case of very small $\omega$ we calculate this correction term by considering the TPSF which acts parallel to the $y$-axis for a fixed amount of time before switching to acting parallel to the $x$-axis. In this flow field the scalar builds up over the first period of the flow and then is sheared perpendicular to the source for the second half of the period. For this flow the scalar rises to the constant value $C_{\max }=\kappa^{-1}$ over the first half period and then reduces to another constant value for the second half of the flow. In this half, for small $\kappa$, the scalar becomes concentrated at $y=0$ in a thin boundary layer, and the solution to this problem is found using matched asymptotic expansions to be $C_{\max } \sim 0.811 \kappa^{-1 / 3}$. This means that as $\omega \rightarrow 0, \overline{C_{\max }}$ $\sim \frac{1}{2}\left(\kappa^{-1}+0.811 \kappa^{-1 / 3}\right)$.

\section{ACKNOWLEDGMENTS}

The authors would like to thank Professor Peter Ashwin for useful discussions in the writing of this paper. This work was supported by the EPSRC under Grant No. EP/D032202/1.

${ }^{1}$ J.-L. Thiffeault, C. R. Doering, and J. D. Gibbon, “A bound on mixing efficiency for the advection-diffusion equation," J. Fluid Mech. 521, 105 (2004).

${ }^{2}$ C. R. Doering and J.-L. Thiffeault, "Multiscale mixing efficiencies for steady sources," Phys. Rev. E 74, 025301 (2006).

${ }^{3}$ T. Tirabassi, "Analytical air pollution advection and diffusion models," Water, Air, Soil Pollut. 47, 19 (1989). 
${ }^{4}$ S. Cerbelli, A. Adrover, and M. Giona, "Enhanced diffusion regimes in bounded chaotic flows," Phys. Lett. A 312, 355 (2003).

${ }^{5}$ M. Giona, A. Adrover, S. Cerbelli, and V. Vitacolonna, "Spectral properties and transport mechanisms of partially chaotic bounded flows in the presence of diffusion," Phys. Rev. Lett. 92, 114101 (2004).

${ }^{6} \mathrm{~S}$. Wiggins and J. M. Ottino, "Foundations of chaotic mixing," Philos. Trans. R. Soc. London, Ser. A 362, 937 (2004).

${ }^{7}$ J. P. Gleeson, "Transient micromixing: Examples of laminar and chaotic stirring," Phys. Fluids 17, 100614 (2005).

${ }^{8} \mathrm{~N}$. Butchart and E. E. Remsberg, "Area of the stratospheric polar vortex as a diagnostic for tracer transport on an isentropic surface," J. Atmos. Sci. 43, 1319 (1986).

${ }^{9} \mathrm{~N}$. Nakamura, "Two-dimensional mixing, edge formation and permeability diagnosed in area coordinate," J. Atmos. Sci. 53, 1524 (1996).

${ }^{10}$ K. B. Winters and E. A. D'Asaro, "Diascalar flux and the rate of fluid mixing," J. Fluid Mech. 317, 179 (1996).

${ }^{11}$ P. Haynes and E. Shuckburgh, "Effective diffusion as a diagnostic of atmospheric transport, 1. Stratosphere," J. Geophys. Res. 105, 22777 (2000).

${ }^{12}$ P. Haynes and E. Shuckburgh, "Effective diffusion as a diagnostic of atmospheric transport, 2. Troposphere and lower stratosphere," J. Geophys. Res. 105, 22795 (2000).
${ }^{13}$ E. Shuckburgh and P. Haynes, "Diagnosing transport and mixing using a tracer-based coordinate system," Phys. Fluids 15, 3342 (2003).

${ }^{14}$ M. R. Turner, A. D. Gilbert, and J. Thuburn, "Effective diffusion of scalar fields in a chaotic flow," Phys. Fluids 20, 107103 (2008).

${ }^{15}$ A. N. Staniforth and J. Côté, "Semi-Lagrangian schemes for atmospheric models: A review," Mon. Weather Rev. 119, 2206 (1991).

${ }^{16}$ A. H. Nayfeh and D. T. Mook, Nonlinear Oscillations (Wiley, New York, 1979).

${ }^{17}$ J.-L. Thiffeault, "Scalar decay in chaotic mixing," in Transport and Mixing in Geophysical Flows, Lecture Notes in Physics Vol. 744 (Springer, Berlin, 2006), p. 3.

${ }^{18} \mathrm{P}$. H. Haynes and J. Vanneste, "What controls the decay of passive scalars in smooth flows?" Phys. Fluids 17, 097103 (2005).

${ }^{19}$ T. M. Antonsen, Jr., Z. Fan, E. Ott, and E. Garcia-Lopez, "The role of chaotic orbits in the determination of power spectra of passive scalars," Phys. Fluids 8, 3094 (1996).

${ }^{20}$ T. A. Shaw, J.-T. Thiffeault, and C. R. Doering, "Stirring up trouble: Multi-scale mixing measures for steady scalar sources," Physica D 231, 143 (2007)

${ }^{21}$ M. Abramowitz and I. A. Stegun, Handbook of Mathematical Functions (Dover, New York, 1964). 